\title{
An Akt-independent pathway for regulation of gluconeogenesis
}

A new pathway for regulation of glucose production in response to insulin and nutrients has been proposed in a study in mice published in Nature Medicine.

The increase in insulin levels that occurs with feeding results in upregulation of Akt in the liver, which in turn inhibits the activity of Foxo1 by phosphorylation. This pathway has been considered the main mechanism by which gluconeogenesis is inhibited in the fed state.

The results of the new study corroborate earlier findings that Akt suppresses Foxo1 activity, as Foxo1-dependent gene expression was constitutively activated in the liver when Akt was deleted in mice. However, the researchers also report that liver-specific deletion of both $A k t$ and Foxo1 in these mice is not associated with glucose intolerance and insulin resistance in the fed state, in contrast with deletion of hepatic Akt only. "This is very compelling evidence that another pathway exists for the regulation of hepatic metabolism," comments Morris Birnbaum, one of the study investigators. When Akt is inactivated, this second, parallel pathway of glucose production in the liver is suppressed by Foxo1.

The researchers plan to investigate this parallel pathway further and are also interested in clarifying the mechanism by which active Foxol in the liver blocks the pathway. "Further down the line we would like to understand how Foxo1 is activated in insulin-resistant states, as our data suggest that this process is not due exclusively to decreased Akt phosphorylation," concludes Birnbaum.

Joana Osório

Original article Lu, M. et al. Insulin regulates liver metabolism in vivo in the absence of hepatic Akt and Foxo1. Nat. Med. doi: 10.1038/nm.2686 